\title{
The Improvement on the Boundedness and Norm of a Class of Integral Operators on $L^{p}$ Space
}

\author{
Lifang Zhou and Jin Lu \\ Department of Mathematics, Huzhou University, Huzhou, Zhejiang 313000, China \\ Correspondence should be addressed to Lifang Zhou; lfzhou@mail.ustc.edu.cn \\ Received 14 July 2014; Accepted 27 August 2014 \\ Academic Editor: Janusz Matkowski
}

Copyright (C) 2015 L. Zhou and J. Lu. This is an open access article distributed under the Creative Commons Attribution License, which permits unrestricted use, distribution, and reproduction in any medium, provided the original work is properly cited.

We prove the condition " $c$ is neither 0 nor a negative integer" can be dropped on the boundedness of a class of integral operators $S_{a, b, c}$ on $L^{p}$ space, which improves the result by Krues and Zhu. Besides, the exact norm of $S_{a, b, c}$ on $L^{p}$ space is also obtained under the assumption $c=n+1+a+b$.

\section{Introduction}

Let $\mathbb{B}_{n}$ be the open unit ball in the complex space $\mathbb{C}^{n}$. The measure,

$$
d \nu_{t}=\left(1-|z|^{2}\right)^{t} d \nu(z)
$$

denotes the weighted Lebesgue measure on $\mathbb{B}_{n}$, where $t$ is real parameter and $v$ is the normalized Lebesgue measure on $\mathbb{B}_{n}$ such that $v\left(\mathbb{B}_{n}\right)=1$. It is easy to know $d v_{t}$ is finite if and only if $t>-1$. Suppose $1 \leq p<\infty$; to simplify the notation, we write $L_{t}^{p}:=L^{p}\left(\mathbb{B}_{n}, v_{t}\right)$ for the weighted $L^{p}$-space under the measure $v_{t}$ on $\mathbb{B}_{n}$ and $L^{p}:=L_{0}^{p}$ for the usual $L^{p}$-space under the measure $\nu$.

Suppose $a, b, c$ are real numbers, and a class of integral operators is defined by

$$
S_{a, b, c} f(z)=\left(1-|z|^{2}\right)^{a} \int_{\mathbb{B}_{n}} \frac{\left(1-|w|^{2}\right)^{b}}{|1-\langle z, w\rangle|^{c}} f(w) d \nu(w)
$$

The class of integral operators is introduced by Kures and Zhu [1]. And it is closely related to "maximal Bergman projection" and Berezin transform. In fact, the boundedness of Bergman projection on $L_{\alpha}^{p}$ comes from the boundedness of the operator

$$
\begin{array}{r}
P_{\alpha}^{\sharp} f(z)=\frac{\Gamma(n+\alpha+1)}{n ! \Gamma(\alpha+1)} \int_{\mathbb{B}_{n}} \frac{f(w)}{|1-\langle z, w\rangle|^{n+1+\alpha}} d v_{\alpha}(w), \\
\alpha>-1,
\end{array}
$$

on $L_{\alpha}^{p}$; see [2]. Therefore, we can call $P_{\alpha}^{\sharp}$ by "maximal Bergman projection," which is the particular case of $S_{a, b, c}$. Berezin transforms, whatever the case of the unit disk [3, page $141]$ or the case of unit ball ([4, page 76], [5, page 383]), are all concluded in the form of $S_{a, b, c}$ with special $a, b, c$.

In [1], Krues and Zhu gave the sufficient and necessary conditions of the boundedness of operator $S_{a, b, c}$.

Theorem A (see [1]). Suppose $c$ is neither 0 nor a negative integer.

(1) The operator $S_{a, b, c}$ is bounded on $L_{t}^{p}(1<p<\infty)$ if and only if $-p a<t+1<p(b+1), c \leq n+1+a+b$.

(2) The operator $S_{a, b, c}$ is bounded on $L_{t}^{1}$ if and only if $-a<$ $t+1<b+1, c=n+1+a+b$ or $-a<t+1 \leq b+1, c<$ $n+1+a+b$.

The main purposes of this note contain two parts. One part is to prove the condition " $c$ is neither 0 nor a negative integer" in Theorem A can be removed; see Section 3. 
The other part is to give the accurate norm of the operator $S_{a, b, c}$ on $L_{t}^{p}$ under the assumption $c=n+1+a+b$, which can be seen from the following two theorems.

Theorem 1. Suppose $c=n+1+a+b$. If $1 \leq p<\infty$ and $-p a<t+1<p(b+1)$, then

$$
\left\|S_{a, b, c}\right\|_{L_{t}^{p} \rightarrow L_{t}^{p}}=\frac{n ! \Gamma(a+(t+1) / p) \Gamma(b+1-(t+1) / p)}{\Gamma^{2}((n+1+a+b) / 2)} .
$$

Else, we also give the sufficient and necessary conditions of the operator $S_{a, b, c}$ on $L^{\infty}$ and the accurate norm under $c=n+1+a+b$ of this case, where $L^{\infty}$ denotes the set of all essentially bounded and measurable functions under the measure $v_{t}$ on $\mathbb{B}_{n}$.

Theorem 2. The operator $S_{a, b, c}$ is bounded on $L^{\infty}$ if and only if $a>0, b>-1$, and $c=n+1+a+b$ or $a \geq 0, b>-1$, and $c<n+1+a+b$. Moreover, when $c=n+1+a+b$, we have

$$
\left\|S_{a, b, c}\right\|_{L^{\infty} \rightarrow L^{\infty}}=\frac{n ! \Gamma(a) \Gamma(1+b)}{\Gamma^{2}((n+1+a+b) / 2)} .
$$

Notice $S_{a, b, c}$ is the generalization of "maximal Bergman projection" and Berezin transform which was first introduced by Berezin [6]. The boundedness of Berezin transform of $f \in L^{1}(\mathbb{D})$ is a well-known fact; see [7, Proposition 2.2]. But the norm of it was not calculated out until 2008 by Dostanić; see [ 8 , Corollary 2]. Recently, the result by Dostanić has been extended to several complex variables in [9, Theorem 1.1]. Thus, Theorems 1 and 2 promote the main results in $[8,9]$. And they also imply the following corollary.

Corollary 3. Suppose $1 \leq p<\infty, \alpha>-1$, and the norm of $P_{\alpha}^{\#}$ on $L_{\alpha}^{p}$ can be

$$
\begin{aligned}
\left\|P_{\alpha}^{\sharp}\right\|_{L_{\alpha}^{p} \rightarrow L_{\alpha}^{p}} & \\
& =\frac{\Gamma((\alpha+1) / p) \Gamma((\alpha+1)-(\alpha+1) / p) \Gamma(n+\alpha+1)}{\Gamma^{2}((n+1+\alpha) / 2) \Gamma(\alpha+1)},
\end{aligned}
$$

which implies $\left\|P_{\alpha}^{\sharp}\right\|_{L_{\alpha}^{p} \rightarrow L_{\alpha}^{p}}$ grows at most like $(\alpha+1)^{-1}$ as $\alpha \rightarrow$ -1 .

Next, we will see that the boundedness of an operator called Berezin-type transform on $L_{t}^{p}$ can also be obtained from our main results. The Berezin-type transform is defined by

$$
\begin{aligned}
& \mathscr{B}_{k, \alpha, \beta} f(z) \\
& =C_{k, \alpha, \beta} \\
& \quad \times \int_{\mathbb{B}_{n}} \frac{\left(1-|z|^{2}\right)^{n+\alpha+\beta+k+1}\left(1-|w|^{2}\right)^{k}}{(1-\langle z, w\rangle)^{n+\alpha+k+1}(1-\langle w, z\rangle)^{n+\beta+k+1}} f(w) d \nu(w),
\end{aligned}
$$

where

$$
C_{k, \alpha, \beta}=\frac{\Gamma(n+\alpha+k+1) \Gamma(n+\beta+k+1)}{\Gamma(n+1) \Gamma(k+1) \Gamma(n+\alpha+\beta+k+1)},
$$

and $n+\alpha+\beta>0, n+\alpha>0, n+\beta>0$, and $k>$ -1 . The transform was introduced by Li and Liu [10] when they discuss whether the mean-value property implies $(\alpha, \beta)$ harmonicity for integrable functions on the unit ball in $\mathbb{C}^{n}$. Notice that

$$
\left|\mathscr{B}_{k, \alpha, \beta} f(z)\right| \leq C_{k, \alpha, \beta} S_{a, b, c}|f|(z)
$$

with $a=n+\alpha+\beta+k+1, b=k$, and $c=n+1+a+b$. And $\mathscr{B}_{k, \alpha, \alpha} f(z)=C_{k, \alpha, \alpha} S_{a, b, c} f(z)$ as $\alpha=\beta$. Therefore, the boundedness of Berezin-type transform $\mathscr{B}_{k, \alpha, \beta}$ on $L_{t}^{p}$ comes from the boundedness of the operator $S_{a, b, c}$ on $L_{t}^{p}$. Thus, we have the following result, which extends Propositions 3.3 and 3.4 in [10] combining the fact of Lemma 2.4 in [10] therein.

Corollary 4. If $1 \leq p<\infty$ such that $-p(n+\alpha+\beta+k+1)<$ $t+1<p(k+1)$, then the Berezin-type $\mathscr{B}_{k, \alpha, \beta}$ is bounded on $L_{t}^{p}$ and

$$
\left\|\mathscr{B}_{k, \alpha, \beta}\right\|_{L_{t}^{p} \rightarrow L_{t}^{p}} \leq \lambda_{k, \alpha, \beta, p} \frac{\Gamma(n+\alpha+k+1) \Gamma(n+\beta+k+1)}{\Gamma^{2}(n+1+(\alpha+\beta) / 2+k)},
$$

where

$$
\begin{aligned}
& \lambda_{k, \alpha, \beta, p} \\
& =\frac{\Gamma(n+\alpha+\beta+k+1+(t+1) / p) \Gamma(k+1-(t+1) / p)}{\Gamma(n+\alpha+\beta+k+1) \Gamma(k+1)} .
\end{aligned}
$$

Moreover, the Berezin-type transform is bounded on $L^{\infty}$, and

$$
\left\|\mathscr{B}_{k, \alpha, \beta}\right\|_{L^{\infty} \rightarrow L^{\infty}} \leq \frac{\Gamma(n+\alpha+k+1) \Gamma(n+\beta+k+1)}{\Gamma^{2}(n+1+(\alpha+\beta) / 2+k)} .
$$

\section{Preliminaries}

A number of hypergeometric functions will appear throughout. We use the classical notation ${ }_{2} F_{1}(\alpha, \beta ; \gamma ; z)$ to denote

$$
{ }_{2} F_{1}(\alpha, \beta ; \gamma ; z)=\sum_{k=0}^{\infty} \frac{(\alpha)_{k}(\beta)_{k}}{(\gamma)_{k}} \frac{z^{k}}{k !}
$$

with $\gamma \neq 0,-1,-2, \ldots$, where

$$
(\alpha)_{0}=1, \quad(\alpha)_{k}=\alpha(\alpha+1) \cdots(\alpha+k-1) \quad \text { for } k \geq 1 .
$$

And the hypergeometric series in (13) converges absolutely for all the value of $|z|<1$. Moreover, as $|z| \rightarrow 1^{-}$, it is easy to know that

$$
{ }_{2} F_{1}(\alpha, \beta ; \gamma ; z) \approx \begin{cases}1, & \text { if } \gamma-\alpha-\beta>0 ; \\ \log \frac{1}{1-|z|}, & \text { if } \gamma-\alpha-\beta=0 ; \\ (1-|z|)^{\gamma-\alpha-\beta}, & \text { if } \gamma-\alpha-\beta<0,\end{cases}
$$


where $a(z) \approx b(z)$ represents the ratio and $a(z) / b(z)$ has a positive finite limit as $|z| \rightarrow 1^{-}$. Now we list a few formulas for easy reference (see [11, Chapter II]):

$$
\begin{gathered}
{ }_{2} F_{1}(\alpha, \beta ; \gamma ; 1)=\frac{\Gamma(\gamma) \Gamma(\gamma-\alpha-\beta)}{\Gamma(\gamma-\alpha) \Gamma(\gamma-\beta)}, \\
\operatorname{Re}(\gamma-\alpha-\beta)>0, \\
{ }_{2} F_{1}(\alpha, \beta ; \gamma ; z)=(1-z)^{\gamma-\alpha-\beta}{ }_{2} F_{1}(\gamma-\alpha, \gamma-\beta ; \gamma ; z), \\
{ }_{2} F_{1}(\alpha, \beta ; \gamma ; z) \\
=\frac{\Gamma(\gamma)}{\Gamma(\lambda) \Gamma(\gamma-\lambda)} \\
\times \int_{0}^{1} t^{\lambda-1}(1-t)^{\gamma-\lambda-1}{ }_{2} F_{1}(\alpha, \beta ; \lambda ; t z) d t,
\end{gathered}
$$

$\operatorname{Re} \gamma>\operatorname{Re} \lambda>0 ; \quad|\arg (1-z)|<\pi ; \quad z \neq 1$.

Lemma 5. Suppose $\operatorname{Re} \delta>0$ and $\operatorname{Re}(\lambda+\delta-\alpha-\beta)>0$. Then

$$
\begin{array}{r}
\int_{0}^{1} t^{\lambda-1}(1-t)^{\delta-1}{ }_{2} F_{1}(\alpha, \beta ; \lambda ; t) d t \\
=\frac{\Gamma(\lambda) \Gamma(\delta) \Gamma(\lambda+\delta-\alpha-\beta)}{\Gamma(\lambda+\delta-\alpha) \Gamma(\lambda+\delta-\beta)} .
\end{array}
$$

Proof. Note that, under the assumption of the lemma, both sides of (18) are continuous at $z=1$. The lemma then follows by letting $z \rightarrow 1$ in (18) and applying (16).

The following integral formulae concerning the hypergeometric function are significant for our main results. And all these formulae are contained in [12]. Now we list them.

Lemma 6 (see [12, Corollary 2.4]). For $\alpha \in \mathbb{R}$ and $\gamma>-1$, we have

$$
\begin{aligned}
\int_{\mathbb{B}_{n}} \frac{\left(1-|w|^{2}\right)^{\gamma}}{|1-\langle z, w\rangle|^{2 \alpha}} d \nu(w) \\
\quad=\frac{n ! \Gamma(1+\gamma)}{\Gamma(n+1+\gamma)}{ }_{2} F_{1}\left(\alpha, \alpha ; n+1+\gamma ;|z|^{2}\right) .
\end{aligned}
$$

Lemma 6 is also contained implicitly in the proof of Theorem 1.4.10 in [13] (see the formula in page 19, line 5 of [13]).

Lemma 7 (see [12, Corollary 2.5]). Suppose that $\alpha, \beta>0, \gamma \in$ $\mathbb{R}$, and $n+\alpha+\beta-2 \gamma>0$. Then

$$
\begin{gathered}
\int_{\mathbb{B}_{n}}|z|^{2 \beta}\left(1-|z|^{2}\right)^{\alpha-1}\left\{\int_{\mathbb{B}_{n}} \frac{\left(1-|w|^{2}\right)^{\beta-1}}{|1-\langle z, w\rangle|^{2 \gamma}} d \nu(w)\right\} d \nu(z) \\
=\frac{n(n !) \Gamma(\alpha) \Gamma(\beta) \Gamma(n+\alpha+\beta-2 \gamma)}{\Gamma^{2}(n+\alpha+\beta-\gamma)} .
\end{gathered}
$$

Proof. Using Lemma 6 in the inner integral, we have

$$
\begin{aligned}
& \frac{n ! \Gamma(\beta)}{\Gamma(n+\beta)} \int_{\mathbb{B}_{n}}|z|^{2 \beta}\left(1-|z|^{2}\right)^{\alpha-1} \\
& \quad \times{ }_{2} F_{1}\left(\gamma, \gamma ; n+\beta ;|z|^{2}\right) d \nu(z) \\
& =\frac{n(n !) \Gamma(\beta)}{\Gamma(n+\beta)} \int_{0}^{1} r^{n+\beta-1}(1-r)^{\alpha-1} \\
& \quad \times{ }_{2} F_{1}\left(\gamma, \gamma ; n+\beta ;|z|^{2}\right) d r .
\end{aligned}
$$

Then (19) gives the result.

The following result, usually called Schur's test, is a very effective tool in proving the $L^{p}$-boundedness of integral operators. See, for example, [3].

Lemma 8. Suppose that $(X, \mu)$ is a $\sigma$-finite measure space, $K(x, y)$ is a nonnegative measurable function on $X \times X$, and $T$ is the associated integral operator:

$$
T f(x)=\int_{X} K(x, y) f(y) d \mu(y) .
$$

Let $1<p<\infty$ and $1 / p+1 / q=1$. If there exist a positive constant $C$ and a positive measurable function $u$ on $X$ such that

$$
\int_{X} K(x, y) u(y)^{q} d \mu(y) \leq C u(x)^{q},
$$

for almost every $x$ in $X$, and

$$
\int_{X} K(x, y) u(x)^{p} d \mu(x) \leq C u(y)^{p},
$$

for almost every $y$ in $X$, then $T$ is bounded on $L^{p}(X, \mu)$ with $\|T\| \leq C$.

\section{The Improvement}

The section mainly proposes the condition " $c$ is neither 0 nor a negative integer" can be omitted in Theorem A. Notice the condition is only used to give $c \leq n+1+a+b$ while proving the necessity for the boundedness of the operator $S_{a, b, c}$ on $L_{t}^{p}(1 \leq$ $p<\infty)$; see [1, lemma 12]. Now we will give a new proof of the necessity for the boundedness of $S_{a, b, c}$ on $L_{t}^{p}$ in Propositions 9 and 11 to introduce the condition can be put off.

Proposition 9. Suppose the operator $S_{a, b, c}$ is bounded on $L_{t}^{p}(1<p<\infty)$, and then $-p a<t+1<p(b+1), c \leq$ $n+1+a+b$.

Proof. Let $q$ be the number such that $1 / p+1 / q=1$. For any fixed $\epsilon>0$, define

$$
\begin{gathered}
g_{\epsilon}(w)=C_{1}(\epsilon)\left(1-|w|^{2}\right)^{(\epsilon-(t+1)) / p}, \\
h_{\epsilon}(z)=C_{2}(\epsilon)\left(1-|z|^{2}\right)^{(\epsilon-(t+1)) / q}|z|^{2(b+1+(\epsilon-t-1) / p)},
\end{gathered}
$$


where

$$
\begin{gathered}
C_{1}(\epsilon)=\left\{\frac{\Gamma(\epsilon) \Gamma(n+1)}{\Gamma(n+\epsilon)}\right\}^{-1 / p}, \\
C_{2}(\epsilon)=\left\{\frac{n \Gamma(\epsilon) \Gamma(n+q(b+1)+q(\epsilon-(t+1)) / p)}{\Gamma(n+q(b+1)+q(\epsilon-(t+1)) / p+\epsilon)}\right\}^{-1 / q} .
\end{gathered}
$$

Easy calculation shows $\left\|g_{\epsilon}\right\|_{p, t}=\left\|h_{\epsilon}\right\|_{q, t}=1$. Notice the fact

$$
\begin{aligned}
& \left\|S_{a, b, c}\right\|_{L_{t}^{p} \rightarrow L_{t}^{p}} \\
& =\sup _{\substack{\|f\|_{p, t}=1 \\
\|g\|_{q, t}=1}} \\
& \times\left\{\mid \int_{\mathbb{B}_{n}}\left(\int_{\mathbb{B}_{n}}\left(1-|z|^{2}\right)^{a}\right.\right. \\
& \left.\left.\times \frac{\left(1-|w|^{2}\right)^{b-t}}{|1-\langle z, w\rangle|^{c}} f(w) d v_{t}(w)\right) \overline{g(z)} d v_{t}(z) \mid\right\} .
\end{aligned}
$$

Then the boundedness of the operator $S_{a, b, c}$ on $L_{t}^{p}$ leads to the integral

$$
\begin{aligned}
& \mid \int_{\mathbb{B}_{n}}\left\{\int_{\mathbb{B}_{n}} \frac{\left(1-|z|^{2}\right)^{a}\left(1-|w|^{2}\right)^{b-t}}{|1-\langle z, w\rangle|^{c}} g_{\epsilon}(w) d \nu_{t}(w)\right\} \\
& \quad \times \overline{h_{\epsilon}(z)} d \nu_{t}(z) \mid \\
& \leq\left\|S_{a, b, c}\right\|_{L_{t}^{p} \rightarrow L_{t}^{p}}<+\infty .
\end{aligned}
$$

Hence, using Lemma 7 with $\alpha=a+\epsilon / q+(t+1) / p, \beta=$ $b+1+(\epsilon-(t+1)) / p$, and $\gamma=c / 2$, we can conclude that

$$
\begin{gathered}
a+\frac{\epsilon}{q}+\frac{t+1}{p}>0, \quad b+1+\frac{\epsilon-(t+1)}{p}>0, \\
n+1+a+b+\epsilon-c>0 .
\end{gathered}
$$

Then the arbitrariness of $\epsilon$ gives

$$
-p a \leq t+1 \leq p(b+1), \quad c \leq n+1+a+b .
$$

Now, we will give the proof by dividing into the following two cases. equals

When $c=n+1+a+b$, by Lemma 7, the integral in (30)

$$
\begin{aligned}
& \frac{n ! \Gamma(a+(\epsilon / q)+((t+1) / p)) \Gamma(b+1+((\epsilon-(t+1)) / p))}{\Gamma^{2}((n+1+a+b) / 2+\epsilon)} \\
& \quad \times\left\{\frac{\Gamma(n+\epsilon)}{\Gamma(n)}\right\}^{1 / p} \\
& \quad \times\left\{\frac{\Gamma(n+q(b+1)+q(\epsilon-(t+1)) / p+\epsilon)}{\Gamma(n+q(b+1)+q(\epsilon-(t+1)) / p)}\right\}^{1 / q} .
\end{aligned}
$$

Then letting $\epsilon \rightarrow 0^{+}$, by (30), we can know the limits

$$
\begin{aligned}
0 & \leq \lim _{\epsilon \rightarrow 0^{+}} \frac{n ! \Gamma(a+(\epsilon / q)+((t+1) / p)) \Gamma(b+1+((\epsilon-(t+1)) / p))}{\Gamma^{2}((n+1+a+b) / 2+\epsilon)} \\
& \leq\left\|S_{a, b, c}\right\|_{L_{t}^{p} \rightarrow L_{t}^{p .}}
\end{aligned}
$$

Then the boundedness of the operator $S_{a, b, c}$ gives $-p a<t+$ $1<p(b+1)$.

When $c<n+1+a+b$, take the function

$$
f_{\lambda}(z)=\left(1-|z|^{2}\right)^{\lambda}
$$

with $\lambda>a$. The condition (32) implies the function $f_{\lambda} \in L_{t}^{p}$. And using Lemma 6, we have

$$
\begin{aligned}
S_{a, b, c} f_{\lambda}(z)= & \left(1-|z|^{2}\right)^{a} \int_{\mathbb{B}_{n}} \frac{\left(1-|w|^{2}\right)^{b}}{|1-\langle z, w\rangle|^{c}} f_{\lambda}(w) d v(w) \\
= & \left(1-|z|^{2}\right)^{a} \frac{n ! \Gamma(1+b+\lambda)}{\Gamma(n+1+b+\lambda)} \\
& \times{ }_{2} F_{1}\left(\frac{c}{2}, \frac{c}{2} ; n+1+b+\lambda ;|z|^{2}\right) .
\end{aligned}
$$

According to (15), we can obtain that $S_{a, b, c} f_{\lambda}(z) \approx\left(1-|z|^{2}\right)^{a}$. Thus the boundedness of the operator $S_{a, b, c}$ on $L_{t}^{p}\left(\mathbb{B}_{n}\right)$ gives that $p a+t>-1$; that is, $-p a<t+1$. Now we consider the adjoint operator $S_{a, b, c}^{*}$ of the operator $S_{a, b, c}$; that is,

$$
S_{a, b, c}^{*} f(z)=\left(1-|z|^{2}\right)^{b-t} \int_{\mathbb{B}_{n}} \frac{\left(1-|w|^{2}\right)^{a+t}}{|1-\langle z, w\rangle|^{c}} f(w) d v(w) .
$$

The boundedness of $S_{a, b, c}$ on $L_{t}^{p}$ implies the boundedness of $S_{a, b, c}^{*}$ on $L_{t}^{q}$. With the similar discussion above, we can obtain that $q(b-t)+t>-1$; that is, $t+1<p(b+1)$.

When $c=n+1+a+b$, (34) implies the following result.

Corollary 10. Suppose $c=n+1+a+b$ and $1<p<\infty$, $-p a<t+1<p(b+1)$, and then

$$
\left\|S_{a, b, c}\right\|_{L_{t}^{p} \rightarrow L_{t}^{p}} \geq \frac{n ! \Gamma(a+(t+1) / p) \Gamma(b+1-(t+1) / p)}{\Gamma^{2}((n+1+a+b) / 2)} .
$$

Proposition 11. The operator $S_{a, b, c}$ is bounded on $L_{t}^{1}$ if and only if $-a<t+1<b+1, c=n+1+a+b$ or $-a<t+1 \leq$ $b+1, c<n+1+a+b$. And when $c=n+1+a+b$, we have

$$
\left\|S_{a, b, c}\right\|_{L_{t}^{1} \rightarrow L_{t}^{1}}=\frac{n ! \Gamma(1+a+t) \Gamma(b-t)}{\Gamma^{2}((n+1+a+b) / 2)} .
$$

When $c<n+1+a+b,-a<t+1=b+1$, we have

$$
\left\|S_{a, b, c}\right\|_{L_{t}^{1} \rightarrow L_{t}^{1}}=\frac{n ! \Gamma(1+a+b) \Gamma(\sigma)}{\Gamma^{2}((n+1+a+b+\sigma) / 2)},
$$

where $\sigma=(n+1+a+b)-c$. 
Proof. By Lemma 6, we have

$$
\begin{aligned}
\left\|S_{a, b, c}\right\|_{L_{t}^{1} \rightarrow L_{t}^{1}}= & \left\|S_{a, b, c}^{*}\right\|_{L^{\infty} \rightarrow L^{\infty}} \\
= & \sup _{z \in \mathbb{B}_{n}}\left(1-|z|^{2}\right)^{b-t} \int_{\mathbb{B}_{n}} \frac{\left(1-|w|^{2}\right)^{a+t}}{|1-\langle z, w\rangle|^{c}} d \nu(w) \\
= & \frac{n ! \Gamma(1+a+t)}{\Gamma(n+1+a+t)} \sup _{z \in \mathbb{B}_{n}}\left(1-|z|^{2}\right)^{b-t} \\
& \times{ }_{2} F_{1}\left(\frac{c}{2}, \frac{c}{2} ; n+1+a+t ;|z|^{2}\right),
\end{aligned}
$$

where $S_{a, b, c}^{*}$ denotes the adjoint operator of $S_{a, b, c}$. Then, using (15), we can obtain that the operator $S_{a, b, c}$ is bounded on $L_{t}^{1}$ if and only if

$$
\begin{gathered}
1+a+t>0, \\
b-t>0, \\
n+1+a+t-c \geq t-b,
\end{gathered}
$$

or

$$
\begin{gathered}
1+a+t>0, \\
b-t=0, \\
n+1+a+t-c>0,
\end{gathered}
$$

which gives the first part of the proposition.

Now we will give the second part. When $c<n+1+a+$ $b$ and $-a<t+1=b+1$, the hypergeometric function in (41) is increasing since its Taylor coefficients are all positive. Applying (16), we have (40). When $c=n+1+a+b$, (17) gives

$$
\begin{gathered}
{ }_{2} F_{1}\left(\frac{n+1+a+b}{2}, \frac{n+1+a+b}{2} ; n+1+a+t ;|z|^{2}\right) \\
=\left(1-|z|^{2}\right)^{t-b} \\
\quad \times{ }_{2} F_{1}\left(\frac{n+1+a-b}{2}+t, \frac{n+1+a-b}{2}+t ;\right. \\
\left.n+1+a+t ;|z|^{2}\right) .
\end{gathered}
$$

Thus (41), the increase of the last hypergeometric function, and (16) lead to

$$
\begin{aligned}
& \left\|S_{a, b, c}\right\|_{L_{t}^{1} \rightarrow L_{t}^{1}} \\
& =\left\|S_{a, b, c}^{*}\right\|_{L^{\infty} \rightarrow L^{\infty}} \\
& =\frac{n ! \Gamma(1+a+t)}{\Gamma(n+1+a+t)}
\end{aligned}
$$

$$
\begin{aligned}
& \times{ }_{2} F_{1}\left(\frac{n+1+a-b}{2}+t, \frac{n+1+a-b}{2}+t ;\right. \\
& n+1+a+t ; 1) \\
& =\frac{n ! \Gamma(1+a+t) \Gamma(b-t)}{\Gamma^{2}((n+1+a+b) / 2)} .
\end{aligned}
$$

\section{The Proof of Theorems 1 and 2}

Proof of Theorems 1 and 2. Since

$$
\left\|S_{a, b, c}\right\|_{L^{\infty} \rightarrow L^{\infty}}=\sup _{z \in \mathbb{B}_{n}}\left(1-|z|^{2}\right)^{a} \int_{\mathbb{B}_{n}} \frac{\left(1-|w|^{2}\right)^{b}}{|1-\langle z, w\rangle|^{c}} d \nu(w),
$$

therefore Theorem 2 comes out as the same discussion as Proposition 11.

Next, we will concentrate on the proof of Theorem 1 . Remember the hypothesis $c=n+1+a+b$ throughout the following proof. Since (39) gives the case of $p=1$, for the case $1<p<\infty$, Corollary 10 gives the lower bound of $\left\|S_{a, b, c}\right\|_{L_{t}^{p} \rightarrow L_{t}^{p}}$. Thus we only show the fact

$$
\left\|S_{a, b, c}\right\|_{L_{t}^{p} \rightarrow L_{t}^{p}} \leq \frac{n ! \Gamma(a+(t+1) / p) \Gamma(b+1-(t+1) / p)}{\Gamma^{2}((n+1+a+b) / 2)} .
$$

To this end, we will use Schur's test (Lemma 8) with

$$
K(z, w)=\frac{\left(1-|z|^{2}\right)^{a}\left(1-|w|^{2}\right)^{b-t}}{|1-\langle z, w\rangle|^{n+1+a+b}} .
$$

Set

$$
u_{t}(z)=\left(1-|z|^{2}\right)^{-(t+1) /(p q)}
$$

where $q$ is the conjugate exponent of $p$ such that $1 / p+1 / q=1$. It then suffices to show

$$
\begin{gathered}
\left(1-|z|^{2}\right)^{a} \int_{\mathbb{B}_{n}} \frac{\left(1-|w|^{2}\right)^{b-t}}{|1-\langle z, w\rangle|^{n+1+a+b}} u_{t}(w)^{q} d \nu_{t}(w) \\
\quad \leq \frac{n ! \Gamma(a+(t+1) / p) \Gamma(b+1-(t+1) / p)}{\Gamma^{2}((n+1+a+b) / 2)} u_{t}(z)^{q},
\end{gathered}
$$

for all $z \in \mathbb{B}_{n}$, and

$$
\begin{aligned}
& \left(1-|w|^{2}\right)^{b-t} \int_{\mathbb{B}_{n}} \frac{\left(1-|z|^{2}\right)^{a}}{|1-\langle z, w\rangle|^{n+1+a+b}} u_{t}(z)^{p} d v_{t}(z) \\
& \leq \frac{n ! \Gamma(a+(t+1) / p) \Gamma(b+1-(t+1) / p)}{\Gamma^{2}((n+1+a+b) / 2)} u_{t}(w)^{p}
\end{aligned}
$$


for all $w \in \mathbb{B}_{n}$. We only prove (50), since (51) comes from the same way as (50). Applying Lemma 6 and (17), we have

$$
\begin{aligned}
\left(1-|z|^{2}\right)^{a} \int_{\mathbb{B}_{n}} \frac{\left(1-|w|^{2}\right)^{b-t}}{|1-\langle z, w\rangle|^{n+1+a+b}} u_{t}(w)^{q} d v_{t}(w) \\
=\left(1-|z|^{2}\right)^{a} \frac{n ! \Gamma(b+1-(t+1) / p)}{\Gamma(n+b+1-(t+1) / p)} \\
\quad \times{ }_{2} F_{1}\left(\frac{n+1+a+b}{2}, \frac{n+1+a+b}{2} ;\right. \\
=\frac{n ! \Gamma(b+1-(t+1) / p)}{\Gamma(n+b+1-(t+1) / p)}\left(1-|z|^{2}\right)^{-(t+1) / p} \\
\quad \times{ }_{2} F_{1}\left(\frac{n+1+b-a}{2}-\frac{t+1}{p}, \frac{n+1+b-a}{2}\right. \\
\left.\quad-\frac{t+1}{p} ; n+1+b-\frac{t+1}{p} ;|z|^{2}\right) .
\end{aligned}
$$

By (16), the last hypergeometric function is bounded from the above by

$$
\begin{aligned}
{ }_{2} F_{1}( & \frac{n+1+b-a}{2}-\frac{t+1}{p}, \frac{n+1+b-a}{2} \\
& \left.-\frac{t+1}{p} ; n+1+b-\frac{t+1}{p} ; 1\right) \\
= & \frac{\Gamma(n+1+b-(t+1) / p) \Gamma(a+(t+1) / p)}{\Gamma^{2}((n+1+a+b) / 2)},
\end{aligned}
$$

since it is increasing on the interval $[0,1)$. This proves $(50)$, which in turn implies (47). The proof is completed.

\section{Remark}

The topic on the exact norm of an operator is an interesting but difficult problem. In this note, we only give the accurate norm of the generalized operator $S_{a, b, c}$ on $L_{t}^{p}$ under $c=n+$ $1+a+b$. But for other cases, except the particular case (40), we can give an upper bound of $\left\|S_{a, b, c}\right\|_{L_{t}^{p} \rightarrow L_{t}^{p}}$ by Theorem 1 according to the fact

$$
\frac{\left(1-|z|^{2}\right)^{a}\left(1-|w|^{2}\right)^{b-t}}{|1-\langle z, w\rangle|^{c}} \leq \frac{2^{\sigma}\left(1-|z|^{2}\right)^{a}\left(1-|w|^{2}\right)^{b-t}}{|1-\langle z, w\rangle|^{n+1+a+b}}
$$

and a lower bound for one fixed $\epsilon>0$ by (30) and Lemma 7; thus the problem of the norm of other cases may be left as an open problem to consider.

\section{Conflict of Interests}

The authors declare that there is no conflict of interests regarding the publication of this paper.

\section{Acknowledgments}

The authors thank the referee for his/her careful reading and corrections. This work is supported by the National Natural Science Foundation of China (nos. 11271124, 11201141, 11301136, 11261022, and 61473332), the Natural Science Foundation of Zhejiang Province (nos. LQ13A010005 and LY14A010017), and the Scientific Research and Teachers Project of Huzhou Teachers College (nos. KX21058 and RP21028).

\section{References}

[1] O. Kures and K. Zhu, "A class of integral operators on the unit ball of $\mathbb{C}^{n}$," Integral Equations and Operator Theory, vol. 56, no. 1, pp. 71-82, 2006.

[2] K. Zhu, "A sharp norm estimate of the Bergman projection in $L^{p}$ spaces," in Bergman Spaces and Related Topics in Complex Analysis, vol. 404 of Contemporary Mathematics, pp. 195-205, American Mathematical Society, Providence, RI, USA, 2006.

[3] K. Zhu, Operator Theory in Function Spaces, American Mathematical Society, Providence, RI, USA, 2nd edition, 2007.

[4] K. Zhu, Spaces of Holomorphic Functions in the Unit Ball, Springer, New York, NY, USA, 2005.

[5] P. Ahern, M. Flores, and W. Rudin, "An invariant volume-meanvalue property," Journal of Functional Analysis, vol. 111, no. 2, pp. 380-397, 1993.

[6] F. A. Berezin, "Covariant and contravariant symbols of operators," Mathematics of the USSR-Izvestiya, vol. 6, no. 5, pp. 11171151, 1972.

[7] H. Hedenmalm, B. Korenblum, and K. Zhu, Theory of Bergman spaces, Springer, New York, NY, USA, 2000.

[8] M. Dostanić, "Norm of Berezin transform on $L^{P}$ space," Journal d'Analyse Mathématique, vol. 104, pp. 13-23, 2008.

[9] C. Liu and L. Zhou, "On the p-norm of the Berezin transform," Illinois Journal of Mathematics, vol. 56, no. 2, pp. 497-505, 2012.

[10] D. Li and C. Liu, "The mean-value property and $(\alpha, \beta)$ harmonicity," Journal of the Australian Mathematical Society, vol. 91, no. 2, pp. 189-206, 2011.

[11] A. Erdélyi, W. Magnus, F. Oberhettinger, and F. Tricomi, Higher Transcendental Functions, vol. 1, McGraw-Hill, New York, NY, USA, 1953.

[12] C. Liu, "Sharp Forelli-Rudin esitimates and the norm of the Bergman projection," Preprint.

[13] W. Rudin, Function Theory in the Unit Ball of $\mathbb{C}^{n}$, Springer, New York, NY, USA, 2008. 


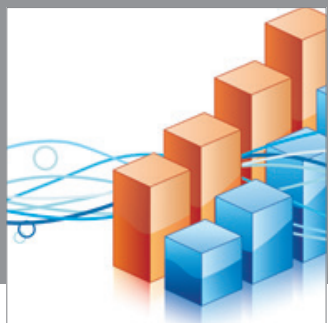

Advances in

Operations Research

mansans

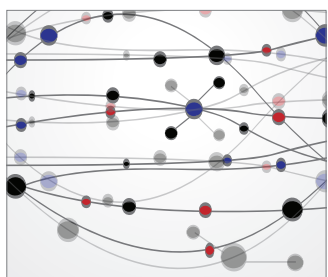

The Scientific World Journal
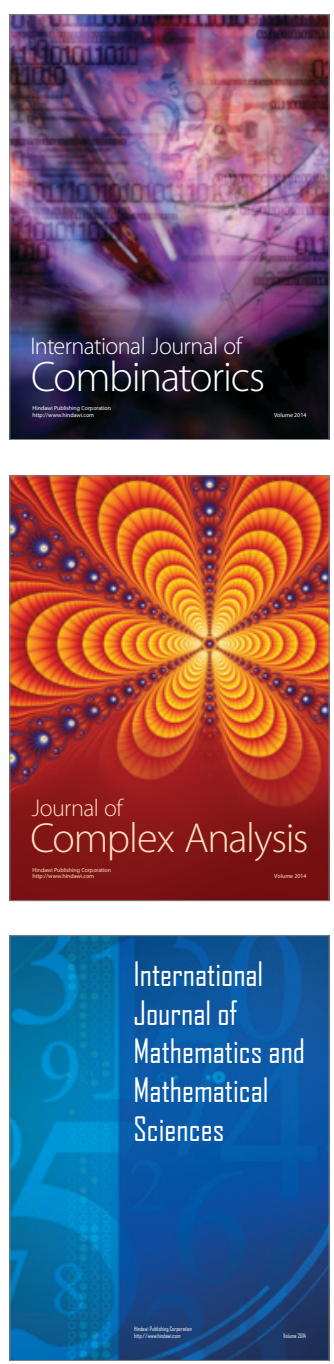
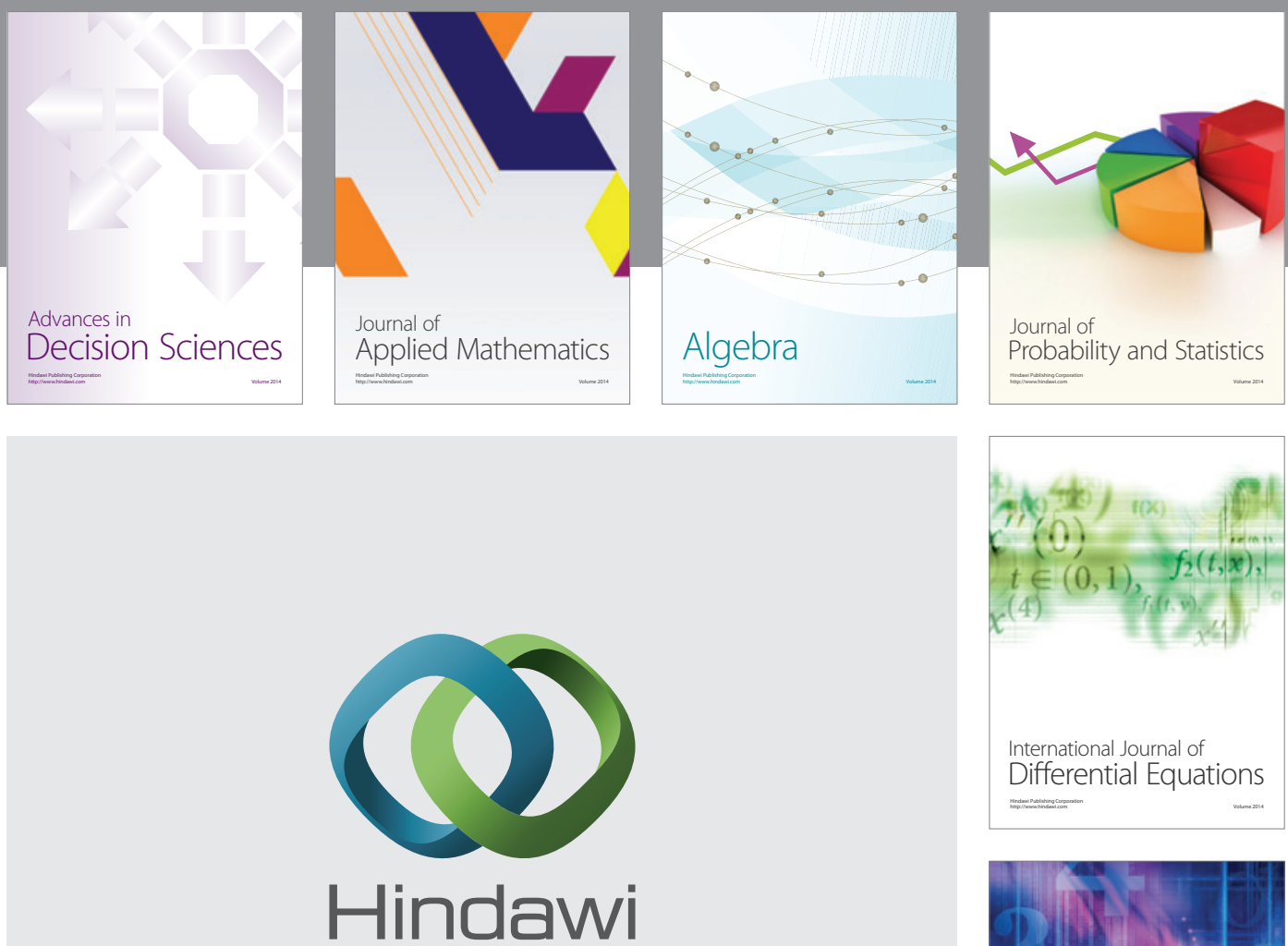

Submit your manuscripts at http://www.hindawi.com
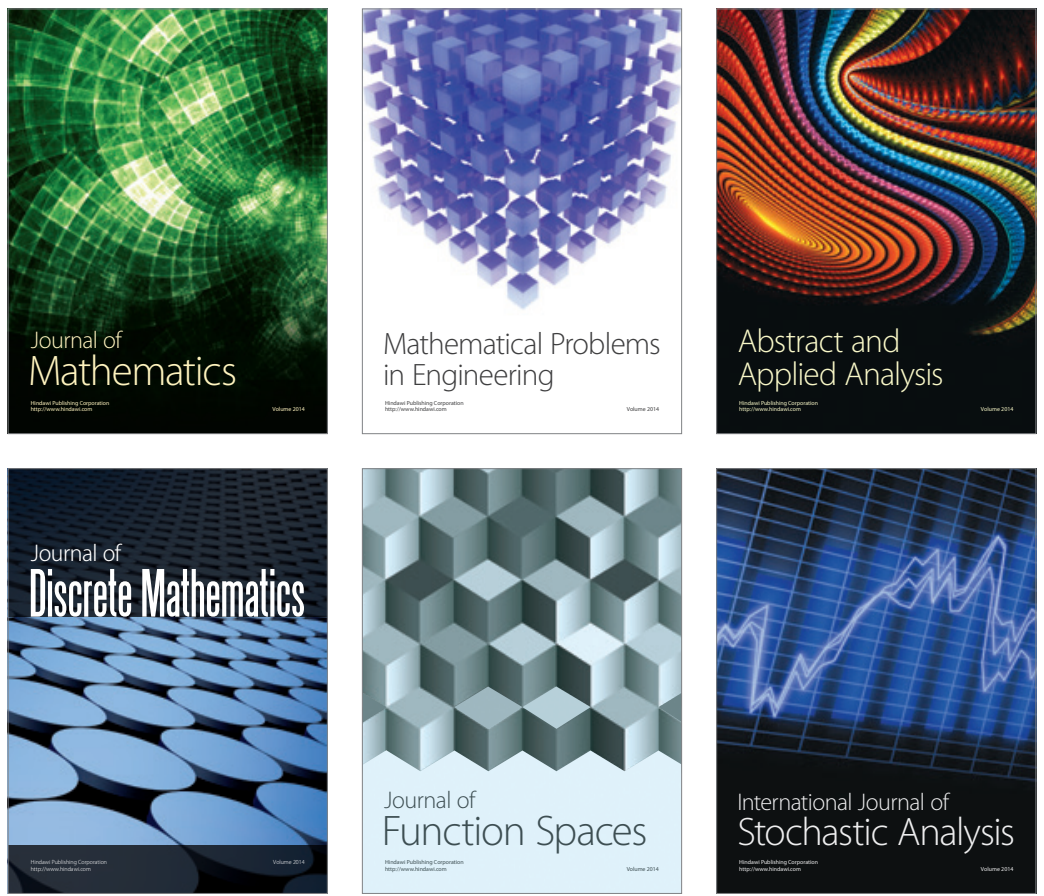

Journal of

Function Spaces

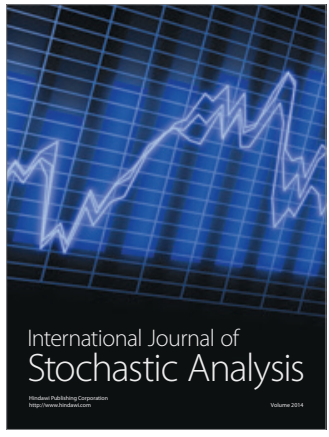

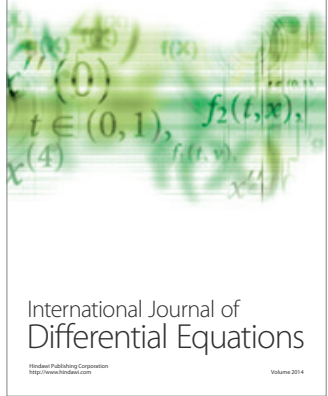
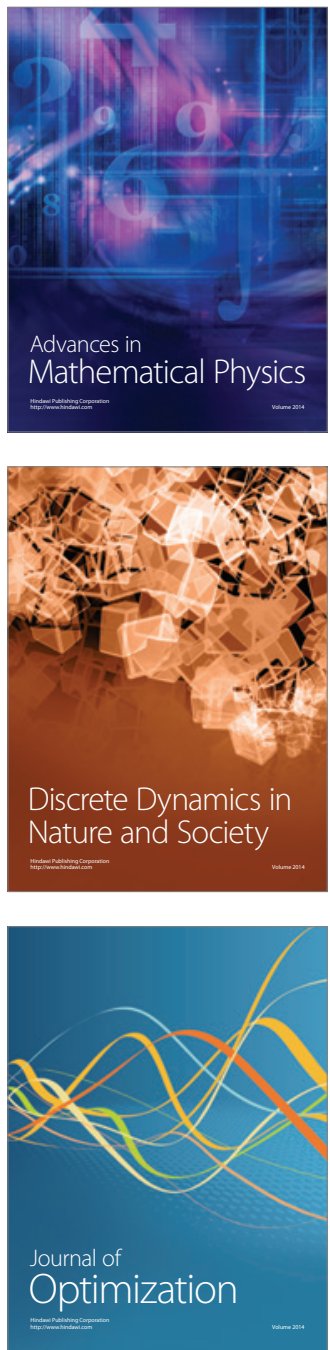